\title{
Electrical Conduction of Superatom Thin Films \\ Composed of Group-V-Metal-Encapsulating Silicon-Cage Nanoclusters
}

\author{
Takaho Yokoyama, ${ }^{\mathrm{a}}$ Tatsuya Chiba, ${ }^{\mathrm{a}}$ Naoyuki Hirata, ${ }^{\mathrm{a}}$ Masahiro Shibuta, ${ }^{\mathrm{b}}$ \\ and Atsushi Nakajima*,a,b \\ ${ }^{a}$ Department of Chemistry, Faculty of Science and Technology, Keio University, 3-14-1 Hiyoshi, \\ Kohoku-ku, Yokohama 223-8522, Japan \\ ${ }^{b}$ Keio Institute of Pure and Applied Science (KiPAS), Keio University, 3-14-1 Hiyoshi, Kohoku-ku, \\ Yokohama 223-8522, Japan \\ *Address correspondence to A. Nakajima \\ Tel: +81-45-566-1712, Fax: +81-45-566-1697, E-mail: nakajima@chem.keio.ac.jp
}

\section{Table of contents}

Full citation of Ref. 14.

Full citation of Ref. 31.

Full citation of Ref. 36 .

Full citation of Ref. 37.

Full citation of Ref. 61.

Figure S1. Au interdigitated patterns on a Si substrate. $\quad$ S3

Figure S2. Mass spectra of $\mathrm{M}^{\mathrm{V}}{ }_{m} \mathrm{Si}_{n}$ binary cluster cations. $\quad \mathrm{S} 3$

Figure S3. Kinetic energy distribution of cluster beam. $\quad$ S4

$\begin{array}{ll}\text { Figure S4. Time evolution of ion current during deposition. } & \text { S4 }\end{array}$

Figure S5. An optical image of deposition spot. $\quad$ S5

Note S1. XPS measurements for chemical evaluation of Ta@Si 16 in assembled films. $\quad$ S5

Figure S6. XPS spectra of Ta@Si $\mathrm{Si}_{16}$ on STO substrate at a bias voltage of -30 V. S6

Figure S7. SEM images of a bare $\mathrm{SiO}_{2}$ surface and $\mathrm{Ag}_{20} \mathrm{NC}$ deposited film. S6

Figure S8. Temperature dependent $I-V$ characteristics of $\mathrm{Nb} @ \mathrm{Si}_{16}$ and Ta@Si $\mathrm{Si}_{16}$ films. $\quad \mathrm{S7}$

Figure S9. $I-V$ characteristic and SEM image of $18.5 \mu \mathrm{C}$ deposited Ta@Si $\mathrm{Si}_{16}$ assembled film. $\quad \mathrm{S} 7$

Table S1. RT conductance, slopes of $\ln G$ vs $T^{-1 / 2}$ and of $\ln W$ vs $\ln T$ plots of $\mathrm{M}^{\mathrm{V}} @ \mathrm{Si} 16$ films. $\quad \mathrm{S} 8$

Figure S10. $I-V$ characteristics of Ta@Si $\mathrm{Si}_{16}$ assembled film with forward and reverse voltage bias. S8

Figure S11. Plots of $\ln G$ versus $T^{-S}$ of $\mathrm{Nb} @ \mathrm{Si}_{16}$ and $\mathrm{Ta} @ \mathrm{Si}_{16}$ assembled films. S9

Figure S12. Plots of $\ln W$ vs $\ln T$ of (a) $\mathrm{Nb} @ \mathrm{Si}_{16}$ and (b) Ta@Si $\mathrm{Si}_{16}$ assembled films. $\quad \mathrm{S} 10$

$\begin{array}{ll}\text { Figure S13. } I-V \text { characteristics at } 0-1 \mathrm{~V} . & \mathrm{S} 10\end{array}$

Figure S14. Molecular orbital diagrams for FK and $f-D_{4 \mathrm{~d}}$ structures of $\mathrm{M}^{\mathrm{V}} @ \mathrm{Si}_{16}{ }^{+} . \quad \mathrm{S} 11$

Note S2. Derivation of parameters of ES-VRH for $\mathrm{M}^{\mathrm{V} @ S i_{16} \text { assembled films. }} \quad \mathrm{S} 11$

Table S2. Parameters of ES-VRH for $\mathrm{M}^{\mathrm{V} @ S i 16 \text { assembled films. }} \quad$ S14

References. $\quad$ S14 


\section{Full citation of Ref. 14.}

Terrill, R. H.; Postlethwaite, T. A.; Chen, C.-H.; Poon, C.-D.; Terzis, A.; Chen, A.; Hutchison, J. E.; Clark, M. R.; Wignall, G.; Londono, J. D.; Superfine, R.; Falvo, M.; Johnson Jr., C. S.; Samulski, E. T.; Murray, R. W. Monolayers in Three Dimensions: NMR, SAXS, Thermal, and Electron Hopping Studies of Alkanethiol Stabilized Gold Clusters. J. Am. Chem. Soc. 1995, 117, 12537-12548.

\section{Full citation of Ref. 31.}

Yuan, P.; Zhang, R.; Selenius, E.; Ruan, P.; Yao, Y.; Zhou, Y.; Malola, S.; Häkkinen, H.; Teo, B. K.; Cao, Y.; Zheng, N. Solvent-Mediated Assembly of Atom-Precise Gold-Silver Nanoclusters to Semiconducting One-Dimensional Materials. Nat. Commun. 2020, 11, 2229.

\section{Full citation of Ref. 36.}

Yang, J.; Zhang, B.; Christodoulides, A. D.; Xu, Q.; Zangiabadi, A.; Peurifoy, S. R.; McGinn, C. K.; Dai, L.; Meirzadeh, E.; Roy, X.; Steigerwald, M. L.; Kymissis, I.; Malen, J. A.; Nuckolls, C. SolutionProcessable Superatomic Thin-Films. J. Am. Chem. Soc. 2019, 141, 10967-10971.

\section{Full citation of Ref. 37.}

Haddon, R. C.; Hebard, A. F.; Rosseinsky, M. J.; Murphy, D. W.; Duclos, S. J.; Lyons, K. B.; Miller, B.; Rosamilia, J. M.; Fleming, R. M.; Kortan, A. R.; Glarum, S. H.; Makhija, A. V.; Muller, A. J.;

Eick, R. H.; Zahurak, S. M.; Tycko, R.; Dabbagh, G.; Thiel, F. A. Conducting Films of $\mathrm{C}_{60}$ and $\mathrm{C}_{70}$ by Alkali-Metal Doping. Nature 1991, 350, 320-322.

\section{Full citation of Ref. 61.}

Frisch, M. J.; Trucks, G. W.; Schlegel, H. B.; Scuseria, G. E.; Robb, M. A.; Cheeseman, J. R.; Scalmani, G.; Barone, V.; Mennucci, B.; Petersson, G. A.; Nakatsuji, H.; Caricato, M.; Li, X.; Hratchian, H. P.; Izmaylov, A. F.; Bloino, J.; Zheng, G.; Sonnenberg, J. L.; Hada, M.; Ehara, M.; Toyota, K.; Fukuda, R.; Hasegawa, J.; Ishida, M.; Nakajima, T.; Honda, Y.; Kitao, O.; Nakai, H.; Vreven, T.; Montgomery, Jr. J. A.; Peralta, J. E.; Ogliaro, F.; Bearpark, M.; Heyd, J. J.; Brothers, E.; Kudin, K. N.; Staroverov, V. N.; Keith, T.; Kobayashi, R.; Normand, J.; Raghavachari, K.; Rendell, A.; Burant, J. C.; Iyengar, S. S.; Tomasi, J.; Cossi, M.; Rega, N.; Millam, J. M.; Klene, M.; Knox, J. E.; Cross, J. B.; Bakken, V.; Adamo, C.; Jaramillo, J.; Gomperts, R.; Stratmann, R. E.; Yazyev, O.; Austin, A. J.; Cammi, R.; Pomelli, C.; Ochterski, J. W.; Martin, R. L.; Morokuma, K.; Zakrzewski, V. G.; Voth, G. A.; Salvador, P.; Dannenberg, J. J.; Dapprich, S.; Daniels, A. D.; Farkas, O.; Foresman, J. B.; Ortiz, J. V.; Cioslowski, J.; Fox, D. J. Gaussian 09, Revision E.01; Gaussian, Inc.: Wallingford, CT, 2013. 


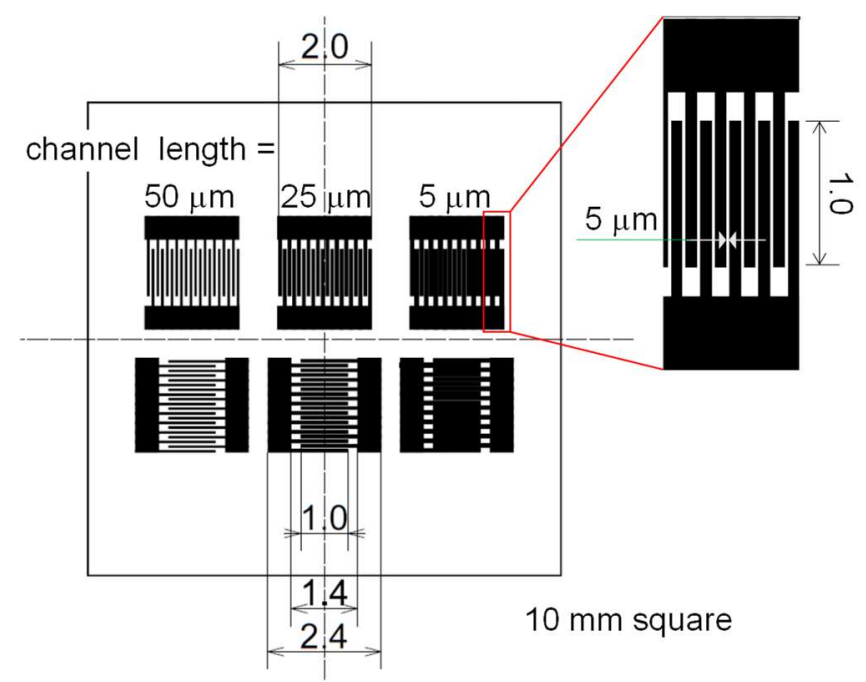

Figure S1. Au interdigitated patterns on a $\mathrm{SiO}_{2}$ (ca. $80 \mathrm{~nm}$ )-layered Si substrate (10 mm square) of doped $n$-Si $(525 \mu \mathrm{m}, 0.001-0.003 \Omega \cdot \mathrm{cm})$ used in this study. Six patterns are fabricated on a Si substrate, and $\mathrm{M}^{\mathrm{V}} @ \mathrm{Si}_{16}$ binary cage superatoms are deposited onto one of the Au interdigitated electrodes (upper right) with a channel length of $5 \mu \mathrm{m}$ and total channel width of $199 \mathrm{~mm}$. By depositing $\mathrm{M}^{\mathrm{V}} @ \mathrm{Si}_{16}$ binary cage superatoms for several hours, a visible spot is formed, as shown in Figure S5.

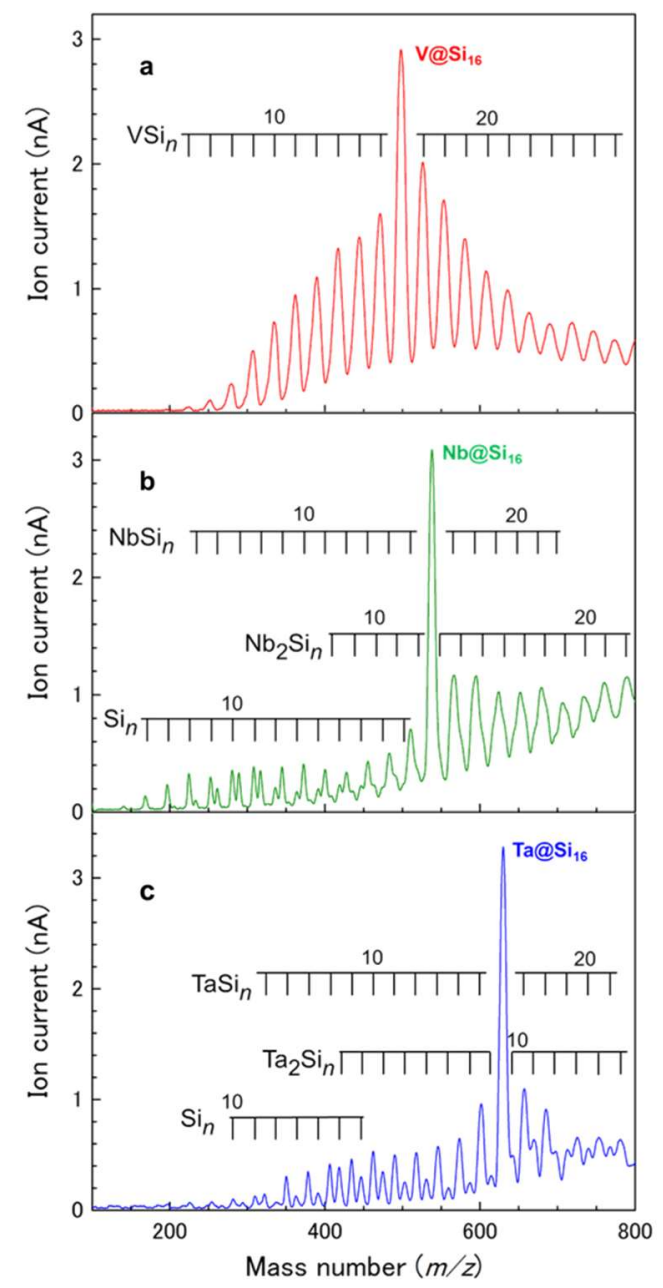

Figure S2. Mass spectra of $\mathrm{M}^{\mathrm{V}}{ }_{m} \mathrm{Si}_{n}$ binary cluster cations for $\mathrm{M}^{\mathrm{V}}=$ (a) $\mathrm{V}$, (b) $\mathrm{Nb}$, and (c) Ta, generated by the high-power impulse magnetron sputtering (HiPIMS) technique. For all $\mathrm{M}^{\mathrm{V}} @ \mathrm{Si}_{16}$ binary cage superatoms, magic number behaviors are observed as selective formation around $3 \mathrm{nA}$ ion currents compared to neighbors. 

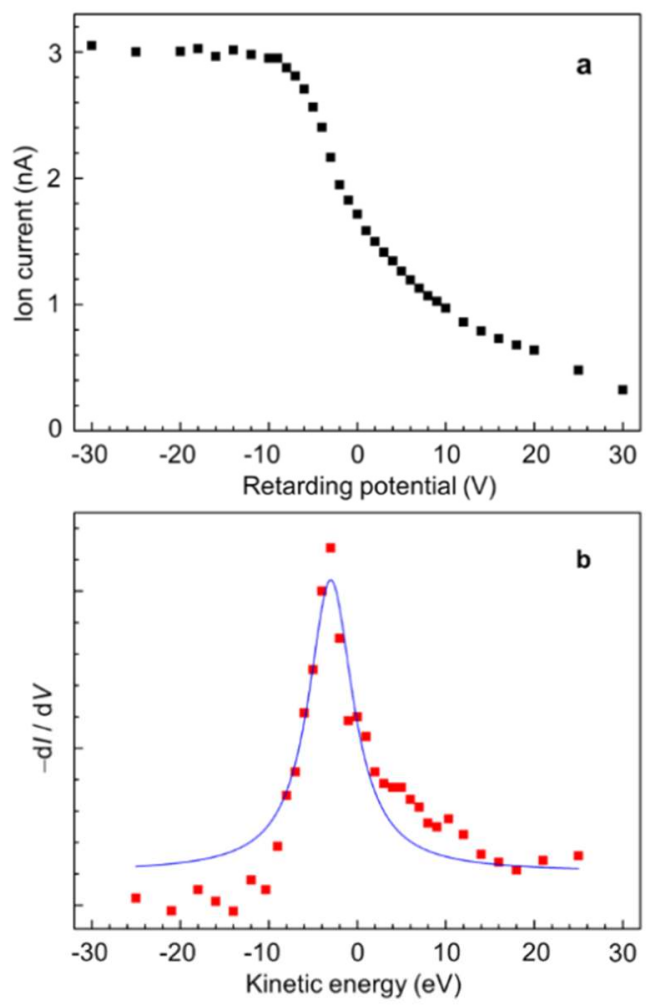

Figure S3. (a) Typical ion current of cationic Ta@ $\mathrm{Si} i_{6}{ }^{+}$binary cage superatoms with variable bias voltages -30 to $+30 \mathrm{~V}$ ). (b) Kinetic energy distribution obtained by the numerical first derivative $(d I / d V)$ : The full width at half-maximum (FWHM) of about $6.2 \mathrm{eV}$ is evaluated by Lorentzian fitting (a blue trace).

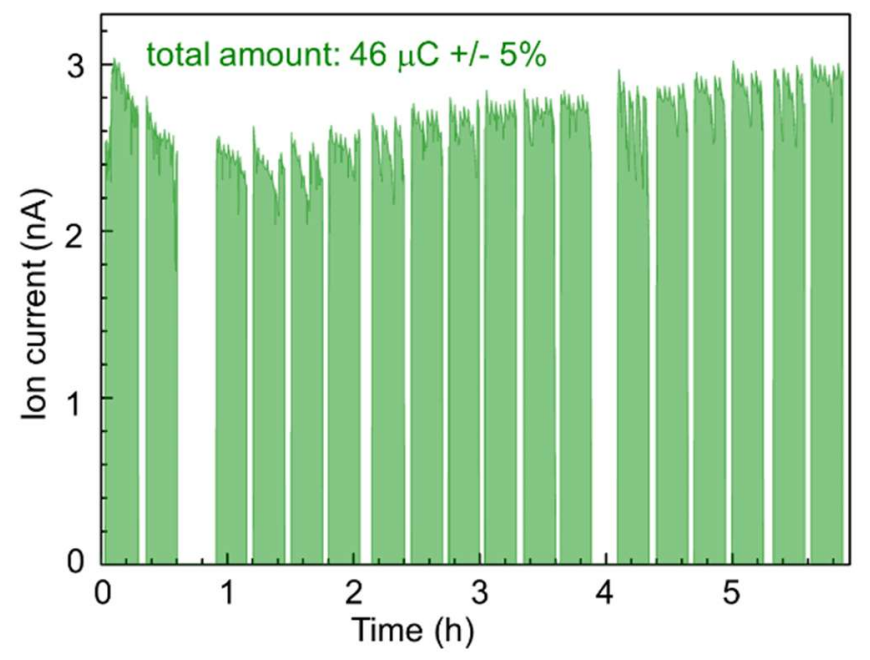

Figure S4. Time evolution of ion currents during the deposition of cationic $\mathrm{Nb} @ \mathrm{Si}_{16}{ }^{+}$binary cage superatoms for $6 \mathrm{~h}$. To maintain constant ion current during the size-selective deposition, magnetron sputtering was tentatively stopped to cool the target surface temperature by filling liquid nitrogen into the cell reservoir every $15 \mathrm{~min}$. The deposition was effectively performed for $4.5 \mathrm{~h}$ in $6 \mathrm{~h}$. The total amount of $\mathrm{M}^{\mathrm{V}} @ \mathrm{Si}_{16}$ binary cage superatoms deposited was about $46 \mu \mathrm{C}+/-5 \%$. This amount corresponds to approximately $100 \mathrm{ML}$. 

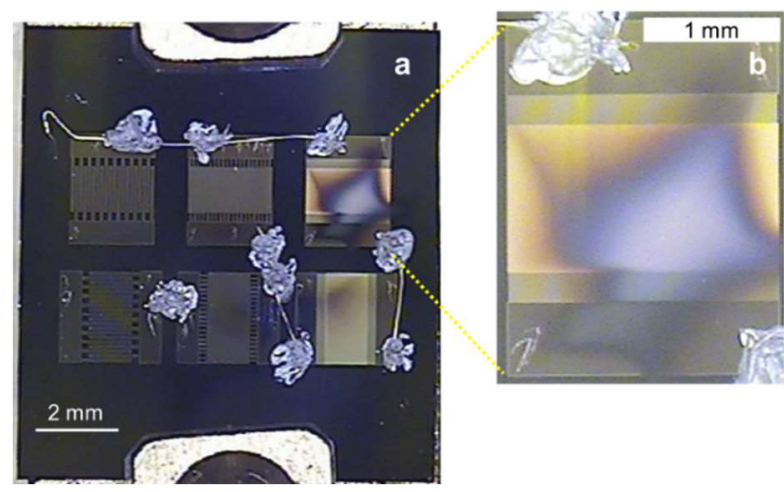

Figure S5. Optical image of the deposition spot of Ta@Si 16 binary cage superatoms on a Si substrate with Au interdigitated electrodes, where one of the six patterns (upper right) was used for the deposition; the channel length was $5 \mu \mathrm{m}$, and the total channel width was $199 \mathrm{~mm}$ (see also Figure S1 for the Au interdigitated patterns).

\section{Note S1. XPS measurements for chemical evaluation of Ta@Si16 in the assembled films}

To examine whether the caged structure of $\mathrm{M}^{\mathrm{V}} @ \mathrm{Si}_{16}$ was maintained after deposition to form an assembled film, X-ray photoelectron spectroscopy (XPS) measurements were conducted using Ta@Si $\mathrm{Si}_{16}$ soft-landed on an oxide substrate of strontium titanate (STO(100); $\mathrm{SrTiO}_{3}, \mathrm{STEP}-\mathrm{SrTiO}_{3} \mathrm{IJ} 100$, Shinkosha Co. Ltd., $0.05 \mathrm{wt} \% \mathrm{Nb}$ dopant), which is conductive and Si-free oxide substrate. Prior to the deposition, the STO surface was cleaned by 2 cycles of $\mathrm{Ar}^{+}$sputtering $(1 \mathrm{kV}, 2 \mu \mathrm{A})$ for 30 min following heating to $450^{\circ} \mathrm{C}$. The XPS spectra were measured using the $\mathrm{Mg} \mathrm{K} \alpha$ line (photon energy $=1253.6 \mathrm{eV}$ ) with an incident angle of $45^{\circ}$ from the surface normal at the base pressure of $2 \times 10^{-8} \mathrm{~Pa}$. Photoelectrons were detected using a hemispherical electron energy analyzer (R3000, VG Scienta) with a take-off angle of $45^{\circ}$. The binding energy was calibrated by the $\mathrm{Au} 4 f_{7 / 2}$ core level. ${ }^{1}$ Since the interfacial layer of $\mathrm{Ta} @ \mathrm{Si}_{16}$ is oxidized by the terminal $\mathrm{O}$ atoms of the STO substrate, ${ }^{2}$ the oxidized components originating from the interfacial layer were evaluated. As a reference, XPS spectra were measured for Si $2 p$ and Ta $4 f$ at predeposited 2.7 ML of Ta@Si 16 . After predeposition, additional 5 ML Ta@Si 16 layers were deposited at a bias voltage of $-30 \mathrm{~V}$, and the XPS spectra were measured for Si $2 p$ and Ta $4 f$. Figure S6 shows the obtained XPS spectra around (a) Si $2 p$ and (b) Ta $4 f$ by subtracting the reference spectrum, in which the obtained XPS spectra were fitted by the single-chemical components of $\mathrm{Si} 2 p_{1 / 2} / \mathrm{Si} 2 p_{3 / 2}$ and Ta $4 f_{5 / 2} / \mathrm{Ta} 4 f_{7 / 2}$ without any oxidation and any unexpected peak broadening.

Since the escape depth of photoelectrons from the substrate is finite, signals originating from $\mathrm{O}$ $2 s$ and $\mathrm{Sr} 4 p$ around the binding energy of $20 \mathrm{eV}$ were weakened with the deposition of Ta@Si $\mathrm{Si}_{16}$, resulting in a negative contribution in the extracted spectra. Peak components of Si $2 p$ and Ta $4 f$ were simulated by a Voigt function with fixed FWHM values of 0.56 and $0.75 \mathrm{eV}$ for Lorentzian and Gaussian, respectively. These values are based on experimental broadening, as determined previously. ${ }^{3}$ The peaks of Si $2 p$ core level are deconvoluted into two components, which are attributed to spin-orbit splitting $\left(2 p_{1 / 2}\right.$ and $\left.2 p_{3 / 2}\right)$ with a known peak separation $(0.608 \mathrm{eV})$ and a branching ratio of $2 J+1(1: 2),{ }^{4}$ while the energy separations and branching ratios of $2 J+1$ are $1.915 \mathrm{eV}$ and 3:4, respectively, for $\mathrm{Ta} 4 f_{5 / 2}$ and $4 f_{7 / 2} .{ }^{5}$ The negative peaks originating from O $2 s$ and $\mathrm{Sr} 4 p$ of the STO substrate were simulated with 
broader FWHM, and the energy separations and branching ratios of $\mathrm{Sr} 4 p$ are $0.7 \mathrm{eV}$ and 1:2, respectively, for $\operatorname{Sr} 2 p_{1 / 2}$ and $2 p_{3 / 2} .{ }^{6}$ As indicated by the orange dotted line, clearly, both Si and Ta atoms are in a uniform chemical environment; in other words, $16 \mathrm{Si}$ atoms are isotropically distributed around a central Ta atom also in the assembled film. Note that when a Ta-Si binary nanocluster forms a noncaged geometric structure, the XPS peaks broaden owing to the inhomogeneous chemical states of Si and Ta atoms, as shown in the XPS spectra of $\mathrm{TaSi}_{8} .^{7}$

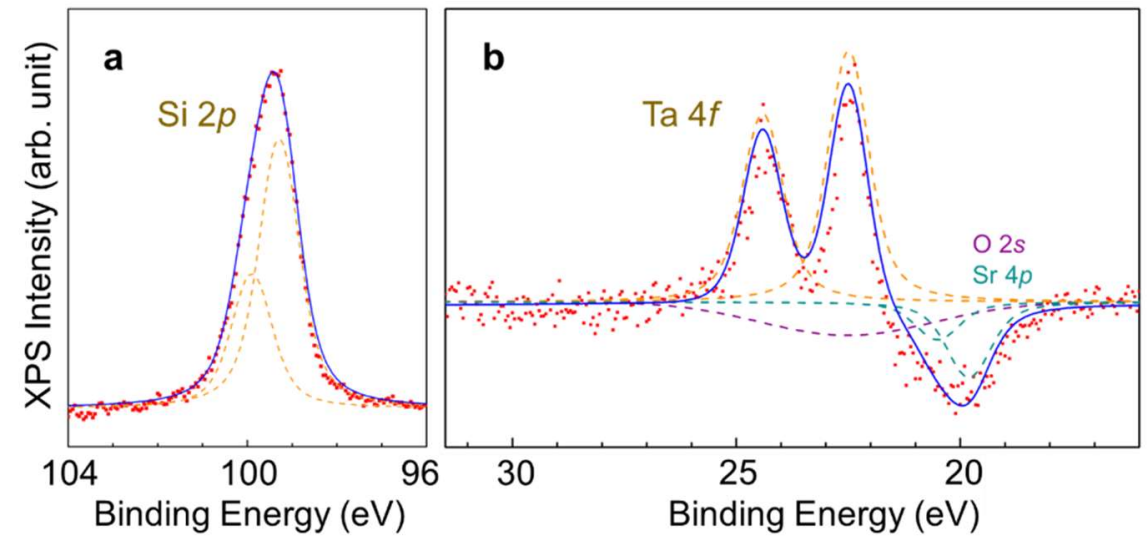

Figure S6. Extracted XPS spectra of Ta@Si $\mathrm{Si}_{16}$ binary cage superatom deposited on the STO with a bias voltage of $-30 \mathrm{~V}$. The fitted results (blue line) and spin-orbit contributions (orange dotted lines) are superimposed in (a) and (b), while the additional components (O $2 s$ and $\mathrm{Sr} 4 p$ ) arising from the STO substrate are indicated in (b) by the purple and turquoise dotted lines, respectively.
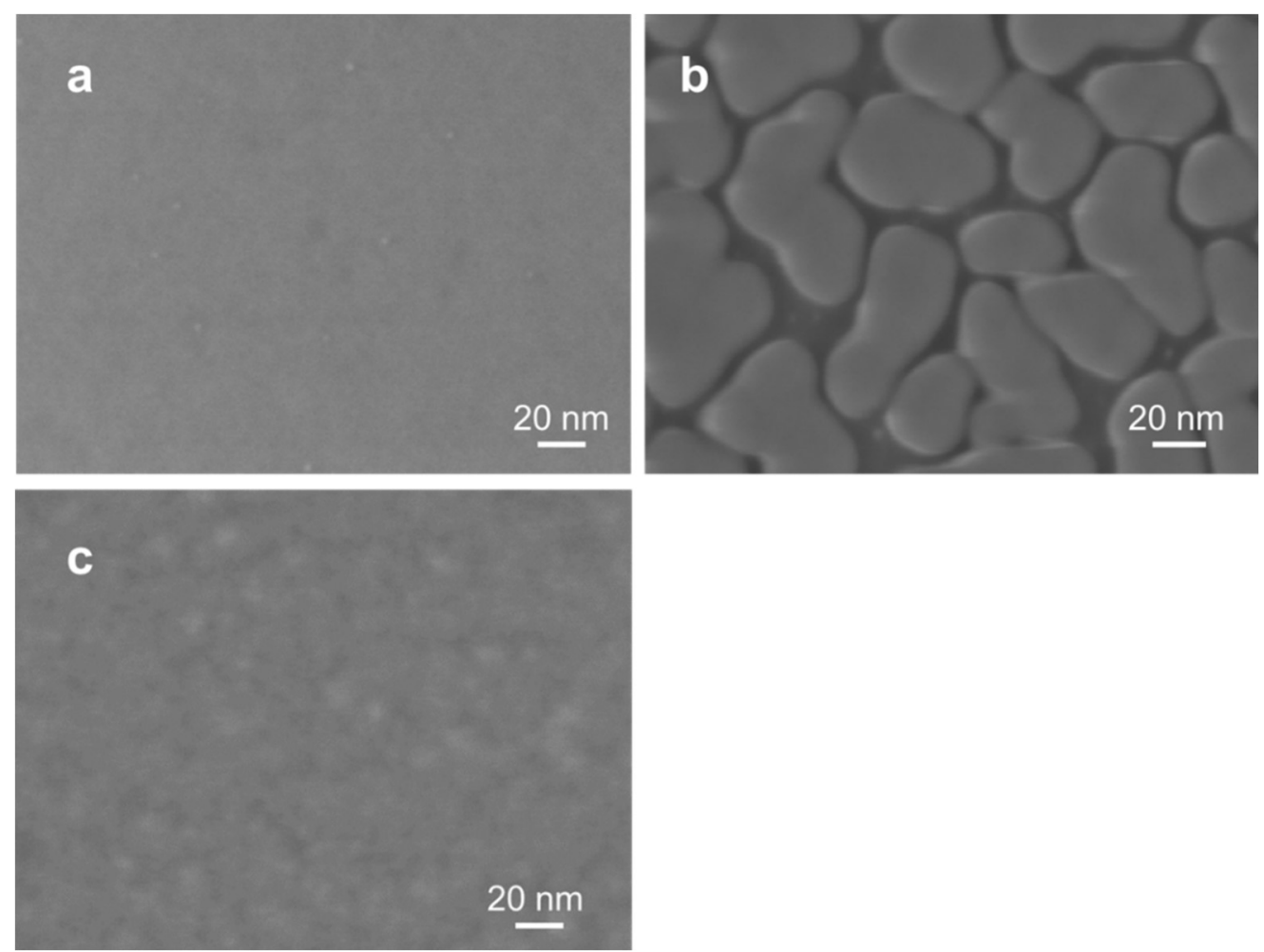

Figure S7. Scanning electron microscopy (SEM) images of (a) the $\mathrm{SiO}_{2}$ surface of a bare substrate without any deposition and (b) a $\mathrm{Ag}_{20} \mathrm{NC}$ deposited film with the same amounts of $\mathrm{M}^{\mathrm{V}} @ \mathrm{Si}_{16}$ binary cage superatom assembled films deposited and (c) Ta@Si 16 binary cage superatom assembled film (same as Figure 1(c) in the main text). 


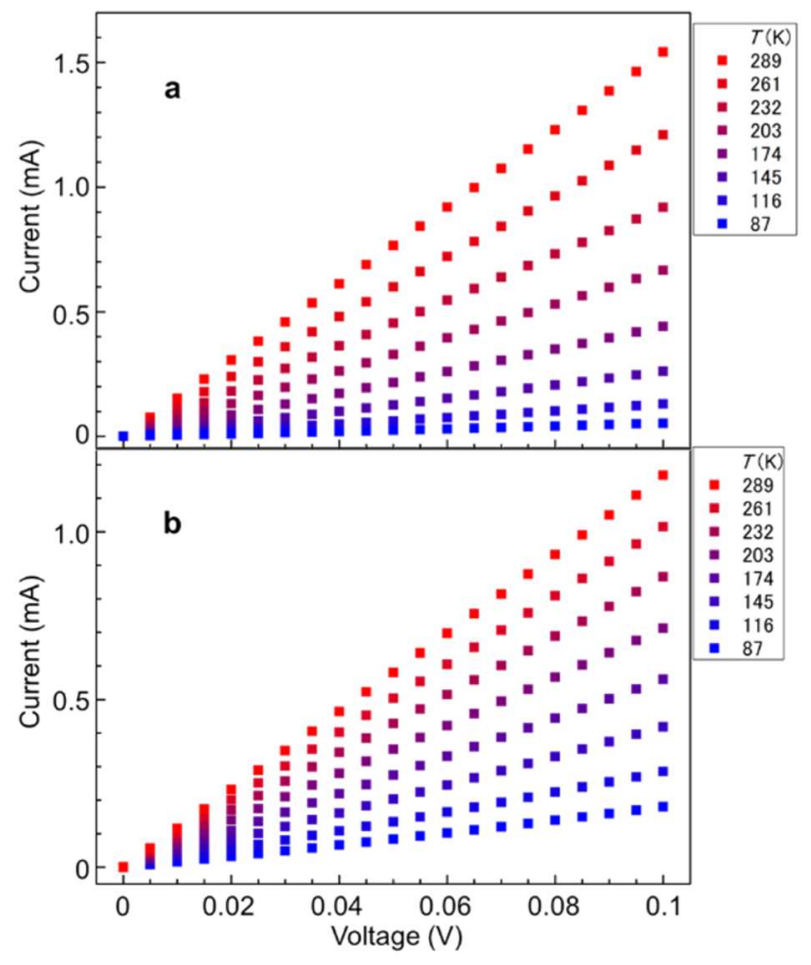

Figure S8. Temperature-dependent $I-V$ characteristics of (a) $\mathrm{Nb} @ \mathrm{Si}_{16}$ and (b) Ta@Si $@$ binary cage superatom assembled films in the range $87-289 \mathrm{~K}$.
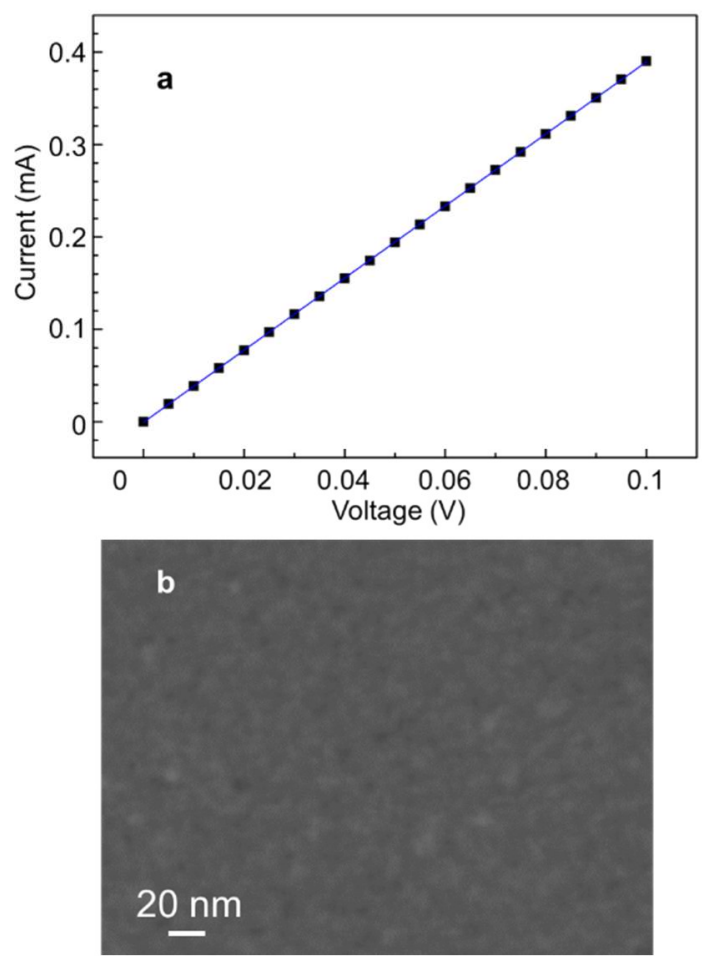

Figure S9. (a) $I-V$ characteristic at RT and (b) SEM image between Au electrodes; on $\mathrm{SiO}_{2}$ of $18.5 \mu \mathrm{C}$ deposited Ta@Si 16 binary cage superatom assembled film. 
Table S1. RT conductance, slopes of the $\ln G$ vs $T^{-1 / 2}$ and $\ln W$ vs $\ln T$ plots of $\mathrm{M}^{\mathrm{V}} @ \mathrm{Si}_{16}$ assembled films for independently fabricated samples.

\begin{tabular}{lcccc}
\hline \hline entry & $\begin{array}{l}\text { conductance at } \\
\mathrm{RT}(\mathrm{mS})\end{array}$ & $\begin{array}{l}\text { Slope of } \\
\text { In } G \text { vs } T^{-1 / 2} \text { plot }\end{array}$ & $\begin{array}{l}\text { Slope of } \\
\text { In } W \text { vs In } T \text { plot }\end{array}$ \\
\hline V@Si 16 & 1 & 15.1 & -60.1 & -0.55 \\
& 2 & 6.0 & -55.1 & -0.37 \\
\hline \multirow{2}{*}{$\mathrm{Nb}_{16 \mathrm{Si}_{16}}$} & 1 & 16.5 & -72.9 & -0.50 \\
& 2 & 7.7 & -67.5 & -0.41 \\
\hline & 1 & 12.3 & -41.0 & -0.42 \\
$\mathrm{Ta@Si}$ & 2 & 41.3 & -41.4 & -0.80 \\
& 3 & 33.8 & -44.4 & -0.51 \\
\hline \hline
\end{tabular}

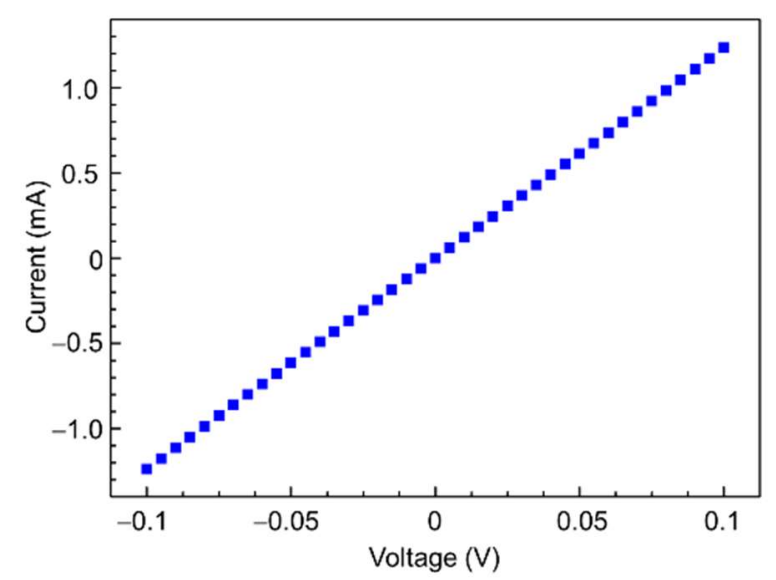

Figure S10. $I-V$ characteristic of Ta@Si 16 assembled film at room temperature with forward and reverse voltage bias. 

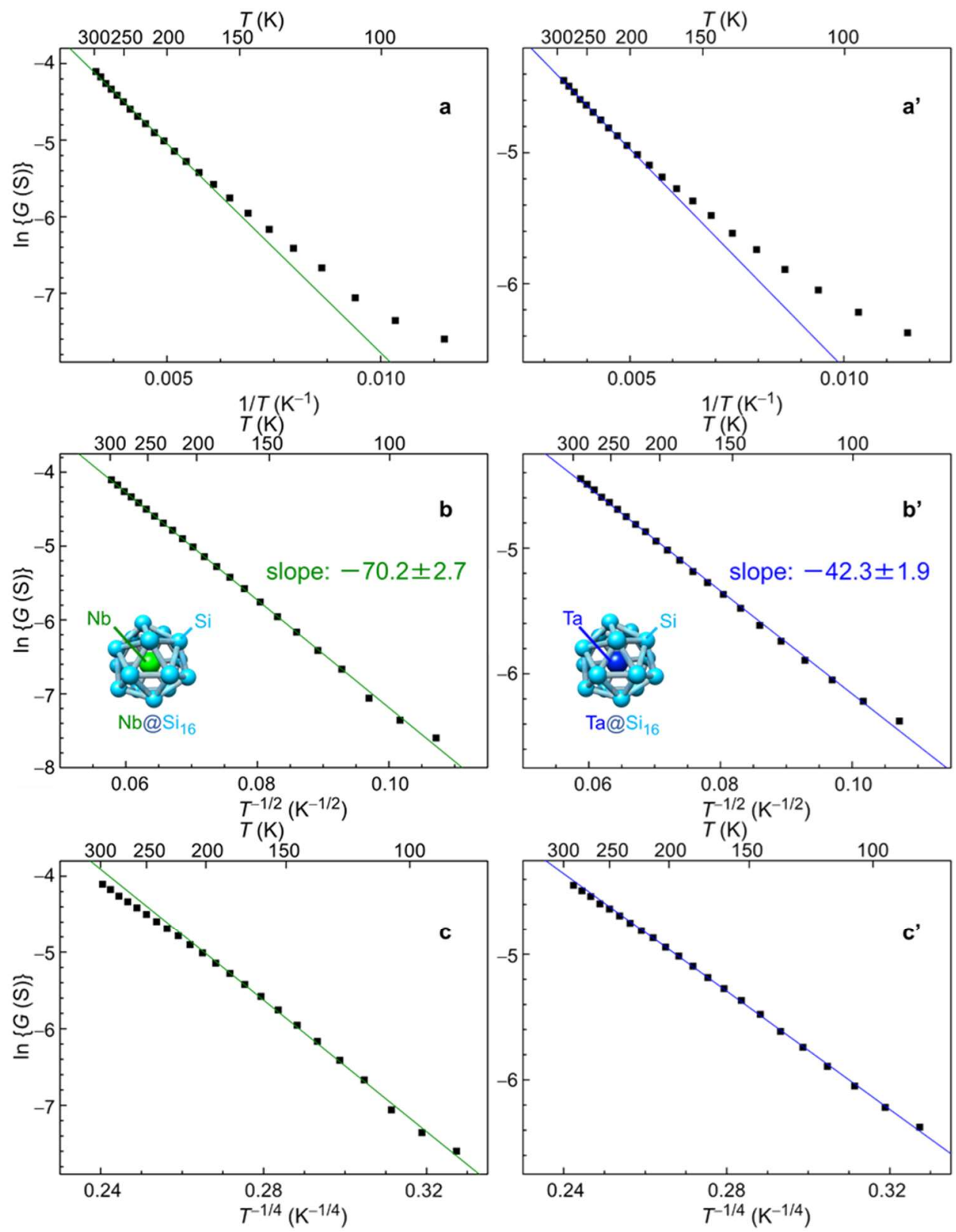

Figure S11. Plots of the natural logarithm of conductance ( $\ln G$ ) versus $T^{-s}$; (a), (b), and (c) show the plots of Nb@Si16, and (a'), (b'), and (c') show the plots for Ta@Si $\mathrm{Si}_{16}$ assembled films. Here, for (a) and (a'), $s=1$; for (b) and (b'), $s=1 / 2$; and for (c) and (c'), $s=1 / 4$ are used. The best fitting with a single straight line is obtained with $s=1 / 2$, while deviations from the linear relationship are observed for $s=$ 1 and $s=1 / 4$. 

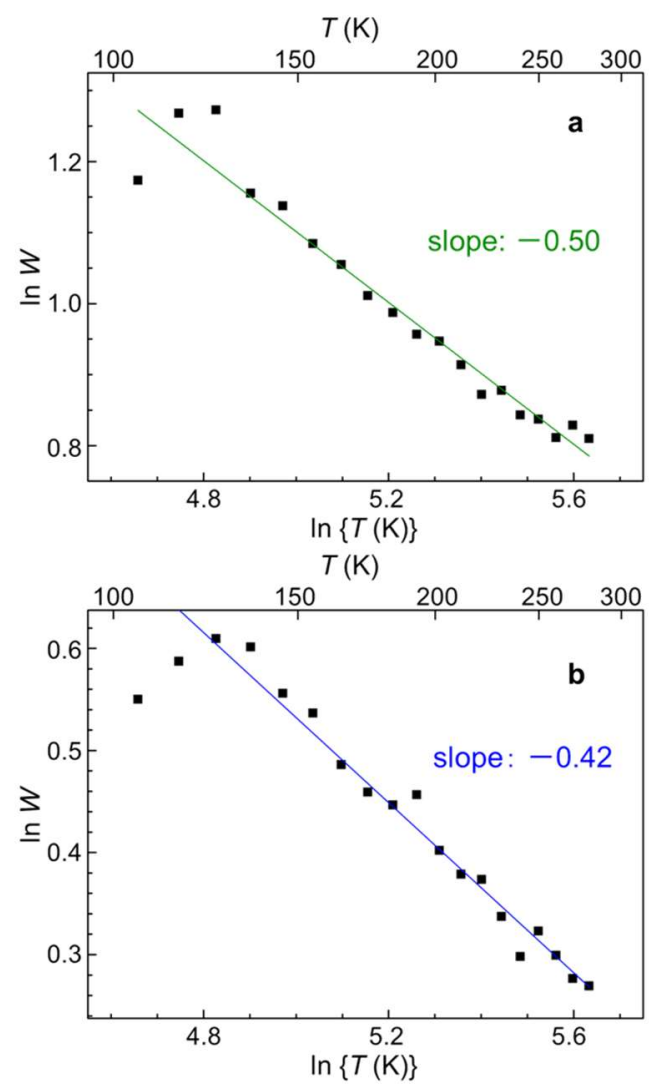

Figure S12. Zabrodskii plots of $\ln W$ vs $\ln T$ for (a) $\mathrm{Nb} @ \mathrm{Si}_{16}$ and (b) Ta@Si $\mathrm{Si}_{16}$ binary cage superatom assembled films. Although the slope in (b) deviates slightly from -0.5 , the ES-VRH conduction mechanism seems reasonable, as seen from the data in Table S1.

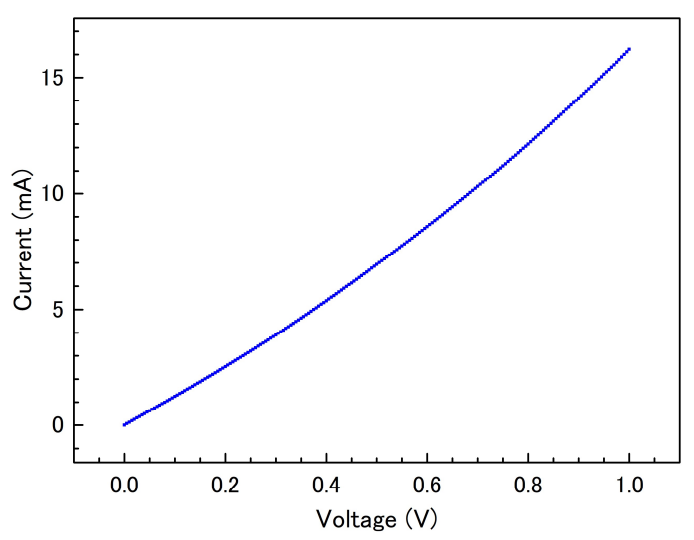

Figure S13. $I-V$ characteristic of the Ta $@ \mathrm{Si}_{16}$ binary cage superatom assembled film at $\mathrm{RT}$ in $0-1 \mathrm{~V}$, showing an increase in current with increasing the electric field. 


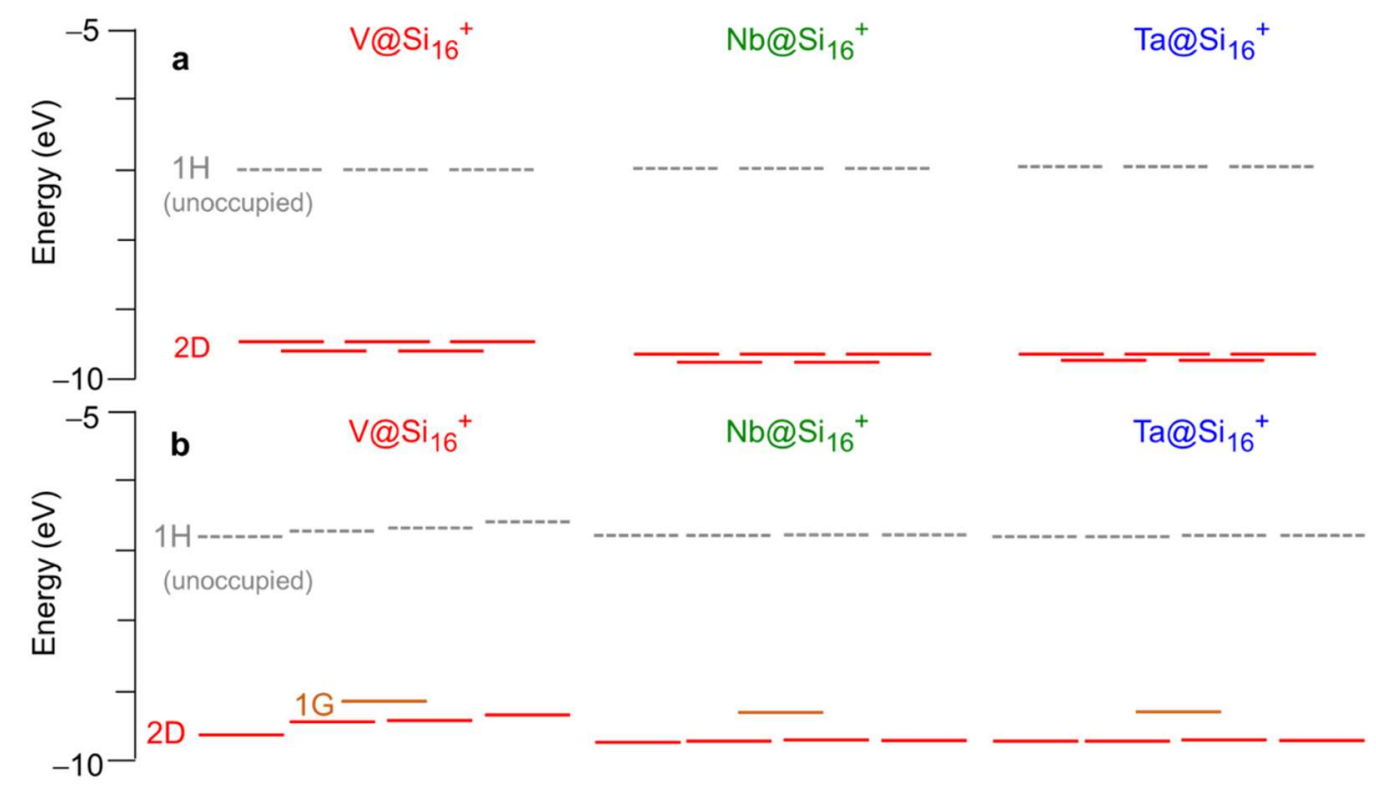

Figure S14. Molecular orbital diagrams around frontier orbitals for (a) FK and (b) $f-D_{4 d}$ structures of $\mathrm{M}^{\mathrm{V}} @ \mathrm{Si}_{16}{ }^{+}$binary cage superatom simulated by DFT calculations. The occupied orbitals are indicated by red and brown, while the unoccupied orbitals are gray. All LUMOs are characterized by superatomic $1 \mathrm{H}$ orbitals, which have multiple nodes.

\section{Note S2. Derivation of parameters of ES-VRH for $\mathbf{M}^{\mathrm{V}} @ \mathrm{Si}_{16}$ assembled films}

In this note, the derivation of some parameters of the ES-VRH model ${ }^{8}$ is described. Some parameters are calculated based on the value of $T_{\mathrm{ES}}$, which is experimentally derived from the square of the slope of the $\ln G$ vs $T^{-1 / 2}$ plot. In the ES-VRH model, the density of states near the Fermi level, $g(\varepsilon)$, is assumed to be split parabolically when the energy difference between Fermi levels $\varepsilon$ is smaller than so-called Coulomb gap $\Delta_{\mathrm{C}}$. In other words, when $|\varepsilon| \leq \Delta_{\mathrm{C}}, g(\varepsilon)$ can be described by the following formula: ${ }^{9}$

$$
g(\varepsilon)=\frac{3^{8} \pi^{2} \varepsilon_{r}{ }^{3} \varepsilon_{0}{ }^{3}}{2^{5} e^{6}} \varepsilon^{2}
$$

When the $|\varepsilon| \geq \Delta_{\mathrm{C}}, g(\varepsilon)$ is a constant value $g_{0}$.

Considering the VRH model, two factors should be addressed: one is the overlap of wavefunctions, and the other is the energy difference between the two hopping sites where the carriers are transferred. The wavefunction is assumed to be determined by the shape of the $s$-orbital, which is represented by $\exp \left(\frac{-r}{\xi}\right)$, where $\xi$ is called localization length that characterizes the shape of assumed wavefunction, and $r$ is the distance from the center of hopping site. Therefore, the overlap of wavefunctions of states $i$ and $j$ with separation distance $r_{i j}$ can be described by $\exp \left(\frac{-r_{i j}}{\xi}\right)$. Since energy levels are different for each $\mathrm{M}^{\mathrm{V}} @ \mathrm{Si}_{16}$ owing to the amorphously assembled conditions, activation energy $\Delta_{i}^{j}=\varepsilon_{j}-\varepsilon_{i}$ is required during hopping transfer from state $i$ to $j$, if the energy of state $j, \varepsilon_{j}$, is higher than that of state $i, \varepsilon_{i}$. Assuming that carriers are transferable between the states 
only in the energy range $|\varepsilon| \leq E_{0}$, a typical value of $\Delta_{i}^{j}$ can be set as $E_{0}$. In addition, $r_{i j}$ can be represented by $\left\{\frac{3}{4 \pi\left(E_{0}\right)}\right\}^{1 / 3}$, where number density, $N\left(E_{0}\right)$, is defined by the following formula.

$$
N\left(E_{0}\right)=\int_{-E_{0}}^{E_{0}} g(\varepsilon) d \varepsilon
$$

Then, electrical conduction, $G$, is proportional as shown in the following formula, wherein the first term in the exponent part represents the overlap of wavefunctions, and the second term represents the activation energy.

$$
G \propto \exp \left[-\frac{3^{1 / 3}}{4^{1 / 3} \pi^{1 / 3}\left\{N\left(E_{0}\right)\right\}^{1 / 3} \xi}-\frac{E_{0}}{k T}\right]
$$

From eqs. (S1) and (S2), $N\left(E_{0}\right)$ can be expressed as a function of $E_{0}$ as shown in eq. (S4), and $G$ can be also regarded as a function of $E_{0}$, as shown in eq. (S5).

$$
\begin{gathered}
N\left(E_{0}\right)=\frac{3^{7} \pi^{2} \varepsilon_{r}{ }^{3} \varepsilon_{0}{ }^{3}}{2^{4} e^{6}} E_{0}{ }^{3} \\
G \propto \exp \left[-\frac{2^{2 / 3} e^{2}}{3^{2} \pi \varepsilon_{r} \varepsilon_{0} \xi} \cdot \frac{1}{E_{0}}-\frac{E_{0}}{k T}\right]
\end{gathered}
$$

Carriers pass through the most efficient path that maximizes the $G$ value; in other words, $E_{0}$ should be the value that minimizes the exponent term in eq. (S5), thereby leading to the following expression:

$$
E_{0}=\frac{2^{1 / 3} e k^{1 / 2} T^{1 / 2}}{3 \pi^{1 / 2} \varepsilon_{r}{ }^{1 / 2} \varepsilon_{0}{ }^{1 / 2} \xi^{1 / 2}}
$$

Since the expression of $E_{0}$ corresponds to the optimal conduction path, $E_{0}$ is called an optimal band. Furthermore, the optimal hopping distance, $\tilde{r}$, can be derived as follows:

$$
\tilde{r}=\frac{2^{1 / 3} e \xi^{1 / 2}}{3 \pi^{1 / 2} \varepsilon_{r}{ }^{1 / 2} \varepsilon_{0}{ }^{1 / 2} k^{1 / 2} T^{1 / 2}}
$$

By substituting $E_{0}$ in eq. (S5) using eq. (S6), the following expression for $G$ can be obtained:

$$
\begin{aligned}
& G \propto \exp \left(-\sqrt{\frac{T_{\mathrm{ES}}}{T}}\right) \\
& \text { where } T_{\mathrm{ES}}=\frac{2^{8 / 3} e^{2}}{3^{2} \pi \varepsilon_{r} \varepsilon_{0} \xi k} \\
& =\frac{2.8 e^{2}}{\kappa \xi k}
\end{aligned}
$$

In the expression of $T_{\mathrm{ES}}, \kappa=4 \pi \varepsilon_{r} \varepsilon_{0}$ is used. As seen clearly from eq. (S8), the slopes of $\ln G$ vs $T^{-1 / 2}$ plot in Figures 2(b) and 3 correspond to $\sqrt{T_{\mathrm{ES}}}$. The squares of the average values of the slope in Figures 2(b) and 3 correspond to the $T_{\mathrm{ES}}$ of $\mathrm{M}^{\mathrm{V}} @ \mathrm{Si}_{16}$ and are listed in Table S2.

Two requirements need to be satisfied when the conduction mechanism is ES-VRH. One is that the optimal hopping distance, $\tilde{r}$, should be larger than the localization length, $\xi$; else, the distance will not match the VRH model wherein carriers pass through the localized electronic states. The other is that the optimal band, $E_{0}$, should be smaller than a Coulomb gap, $\Delta_{C}$; else, the electronic correlation becomes less important, and the alternative conduction mechanism Mott-VRH would be applicable. 
These two requirements are discussed below for all $\mathrm{M}^{\mathrm{V}} @ \mathrm{Si}_{16}$ assembled films to support our claim that the ES-VRH model is reasonably applicable to the results.

From eq. (S9), the localization length, $\xi$, can be derived from $T_{\mathrm{ES}}$ as follows:

$$
\xi=\frac{2^{8 / 3} e^{2}}{3^{2} \pi \varepsilon_{r} \varepsilon_{0} k T_{\mathrm{ES}}}
$$

Using the value of $\varepsilon_{r}=12$, referring to bulk $\mathrm{Si}^{10}{ }^{10} \xi$ can be calculated as tabulated in Table S2. The values of the localization length are a few times larger than the radius of $\mathrm{M}^{\mathrm{V}} @ \mathrm{Si}_{16}$ (ca. $\left.0.45 \mathrm{~nm}\right),{ }^{11}$ implying that domains composed of several $\mathrm{M}^{\mathrm{V}} @ \mathrm{Si}_{16}$ are formed in the assembled films. The optimal length can also be calculated from $T_{\mathrm{ES}}$ as the expression is derived from eqs. (S7) and (S10).

$$
\tilde{r}=\frac{2^{5 / 3} e^{2}}{3^{2} \pi \varepsilon_{r} \varepsilon_{0} k T^{1 / 2} T_{\mathrm{ES}}{ }^{1 / 2}}
$$

The obtained $\tilde{r}$ values of $\mathrm{M}^{\mathrm{V}} @ \mathrm{Si}_{16}$ assembled films are summarized in Table S2 for $T=90$ and $300 \mathrm{~K}$, which are close to the lowest and highest temperatures at which $I-V$ measurements are conducted. The data in Table S2 clearly show that the requirement of $\xi<\tilde{r}$ is satisfied for all temperature ranges and for all $\mathrm{M}^{\mathrm{V}}$. The optimal band, $E_{0}$, derived from eqs. (S6) and (S10) is given by the following formula:

$$
E_{0}=\frac{1}{2} k T^{1 / 2} T_{\mathrm{ES}}^{1 / 2}
$$

$E_{0}$ is calculated for $T=90$ and $300 \mathrm{~K}$ as summarized in Table S2. Since the $E_{0}$ values for $20-30 \mathrm{meV}$ at $T=90 \mathrm{~K}$ and $30-50 \mathrm{meV}$ at $T=300 \mathrm{~K}$ are larger than the thermal energy, $k T, 7.8$ and $26 \mathrm{meV}$ at $T=90$ and $300 \mathrm{~K}, E_{0}$ can reasonably regarded as the typical activation energy for hopping conductivity. Since the density of states, $g(\varepsilon)$, becomes constant $\left(g_{0}\right)$ at $|\varepsilon| \geq \Delta_{\mathrm{C}}, g\left(\Delta_{\mathrm{c}}\right)=g_{0}$ holds, and a Coulomb gap can be described as follows:

$$
\begin{aligned}
& \frac{3^{8} \pi^{2} \varepsilon_{r}{ }^{3} \varepsilon_{0}{ }^{3}}{2^{5} e^{6}} \Delta_{\mathrm{c}}{ }^{2}=g_{0} \\
& \therefore \Delta_{\mathrm{c}}=\frac{2^{5 / 2} e^{3} g_{0}{ }^{1 / 2}}{3^{4} \pi \varepsilon_{r}{ }^{3 / 2} \varepsilon_{0}{ }^{3 / 2}}
\end{aligned}
$$

For $E_{0}<\Delta_{\mathrm{c}}$ to hold, the lowest limit of the density of states near the Fermi level can be estimated.

$$
\begin{gathered}
\frac{1}{2} k T^{1 / 2} T_{\mathrm{ES}}{ }^{1 / 2}<\frac{2^{5 / 2} e^{3} g_{0}{ }^{1 / 2}}{3^{4} \pi \varepsilon_{r}{ }^{3 / 2} \varepsilon_{0}{ }^{3 / 2}} \\
\therefore g_{0}>\frac{3^{8} \pi^{2} \varepsilon_{r}{ }^{3} \varepsilon_{0}{ }^{3} k^{2} T T_{\mathrm{ES}}}{2^{7} e^{6}}
\end{gathered}
$$

For all $\mathrm{M}^{\mathrm{V}} @ \mathrm{Si}_{16}$ assembled films, the ES-VRH model is applicable at least up to $300 \mathrm{~K}$. By substituting $T_{\mathrm{ES}}$ for each of $\mathrm{M}^{\mathrm{V}} @ \mathrm{Si}_{16}$, the lowest limits of $g_{0}$ for each $\mathrm{M}^{\mathrm{V}} @ \mathrm{Si}_{16}$ can be derived.

$$
\begin{aligned}
& g_{0}^{\mathrm{V} @ \mathrm{Si}_{16}}>1.1 \times 10^{21}\left(\mathrm{eV}^{-1} \mathrm{~cm}^{-3}\right) \\
& g_{0}^{\mathrm{Nb} @ \mathrm{Si}_{16}}>1.7 \times 10^{21}\left(\mathrm{eV}^{-1} \mathrm{~cm}^{-3}\right) \\
& g_{0}^{\mathrm{Ta} @ \mathrm{Si}_{16}}>1.2 \times 10^{21}\left(\mathrm{eV}^{-1} \mathrm{~cm}^{-3}\right)
\end{aligned}
$$

The above discussion reasonably shows that the electrical conduction mechanism can be described by the ES-VRH model. 
Table S2. Parameters of the ES-VRH for the $\mathrm{M}^{\mathrm{V}} @ \mathrm{Si}_{16}$ assembled films derived from temperaturedependent $I-V$ characteristics. $\xi$ : localization length, $\tilde{r}$ : optimal hopping distance, and $E_{0}$ : optimal band.

\begin{tabular}{ccccccc}
\hline \hline & $T_{\mathrm{ES}}(\mathrm{K})$ & $\xi(\mathrm{nm})$ & $\begin{array}{c}\tilde{r}(\mathrm{~nm}) \\
\text { at } 90 \mathrm{~K}\end{array}$ & $\begin{array}{c}\tilde{r}(\mathrm{~nm}) \\
\text { at 300 K }\end{array}$ & $\begin{array}{c}E_{0}(\mathrm{meV}) \\
\text { at } 90 \mathrm{~K}\end{array}$ & $\begin{array}{c}E_{0}(\mathrm{meV}) \\
\text { at 300 K }\end{array}$ \\
\hline $\mathrm{V} @ \mathrm{Si}_{16}$ & 3318 & 1.2 & 3.6 & 2.0 & 24 & 43 \\
\hline $\mathrm{Nb@SS_{16 }}$ & 4928 & 0.80 & 2.9 & 1.6 & 29 & 52 \\
\hline $\mathrm{Ta} @ \mathrm{Si}_{16}$ & 1789 & 2.2 & 4.9 & 2.7 & 17 & 32 \\
\hline \hline
\end{tabular}

\section{References}

(1) Seah, M. P.; Gilmore, I. S.; Beamson, G. XPS: Binding Energy Calibration of Electron Spectrometers 5 - Re-Evaluation of the Reference Energies. Surf. Interface Anal. 1998, 26, 642649.

(2) Shibuta, M.; Takano, R.; Nakajima, A. Interfacial Oxidation of Ta-Encapsulating $\mathrm{Si}_{16}$ Cage Superatoms (Ta@Si 16 ) on Strontium Titanate Substrates. J. Phys. Chem. C 2020, 124, 2810828115.

(3) Ohta, T.; Shibuta, M.; Tsunoyama, H.; Negishi, Y.; Eguchi, T.; Nakajima, A. Size and Structure Dependence of Electronic States in Thiolate-Protected Gold Nanoclusters of $\mathrm{Au}_{25}(\mathrm{SR})_{18}$, $\mathrm{Au}_{38}(\mathrm{SR})_{24}$, and $\mathrm{Au}_{144}(\mathrm{SR})_{60}$. J. Phys. Chem. C 2013, 117, 3674-3679.

(4) Rangelov, G.; Fauster, T. Thermally-Induced Evolution of Codeposited Co-Si Layers on Si(100) Surfaces. Surf. Sci. 1996, 365, 403-410.

(5) Riffe, D. M.; Wertheim, G. K. Ta(110) Surface and Subsurface Core-Level Shifts and $4 f_{7 / 2}$ Line Shapes. Phys. Rev. B 1993, 47, 6672-6679.

(6) Tchiomo, A. P. N.; Babu-Geetha, G.; Carleschi, E.; Ngabonziza, P.; Doyle, B. P. Surface Characterization of Clean $\mathrm{SrTiO}_{3}(100)$ Substrates by X-Ray Photoelectron Spectroscopy. Surf. Sci. Spectra 2018, 25, 024001.

(7) Shibuta, M.; Kamoshida, T.; Ohta, T.; Tsunoyama, H.; Nakajima, A. Oxidative Reactivity of Alkali-like Superatoms of Group 5 Metal-Encapsulating $\mathrm{Si}_{16}$ Cage Nanoclusters. Commun. Chem. 2018, 1,50 .

(8) Shklovskii, B. I.; Efros, A. L. Electronic Properties of Doped Semiconductors; Springer: Berlin, 1984.

(9) Adkins, C. J. Conduction in Granular Metals-Variable-Range Hopping in a Coulomb Gap ? J. Phys. Condens. Matter 1989, 1, 1253-1259.

(10) Dunlap, W. C. J.; Watters, R. L. Direct Measurement of the Dielectric Constants of Silicon and Germanium. Phys. Rev. 1953, 92, 1396-1397.

(11) Nakaya, M.; Iwasa, T.; Tsunoyama, H.; Eguchi, T.; Nakajima, A. Formation of a Superatom Monolayer Using Gas-Phase-Synthesized Ta@Si 16 Nanocluster Ions. Nanoscale 2014, 6, 1470214707. 